\title{
The Effect on Children's Attitudes Towards Food Associated With Their Non-Cognitive Skills, and With the Nutrition Knowledge of Their Parents
}

\author{
Tomoko Osera $^{1,2}$, Nobuko Taniguchi ${ }^{3}$, Hiroko Hashimoto ${ }^{1,3}$ \& Nobutaka Kurihara ${ }^{1}$ \\ ${ }^{1}$ Hygiene and Preventive Medicine, Graduate School of Life Science, Kobe Women's University, Kobe, Japan \\ ${ }^{2}$ Department of Nutrition and Health Sciences Faculty of Food and Nutritional Sciences, Toyo University, Japan \\ ${ }^{3}$ Department of Nutrition, Osaka Seikei College, Osaka, Japan \\ Correspondence: Nobutaka Kurihara, Graduate School of Life Science, Kobe Women's University, 2-1 \\ Higashisuma-Aoyama, Suma, Kobe, Japan. Tel: 81-78-737-2417. E-mail: kurihara@suma.kobe-wu.ac.jp
}

Received: April 27, 2018 Accepted: May 29, 2018 Online Published: June 11, 2018

doi:10.5539/jedp.v8n2p54 URL: http://doi.org/10.5539/jedp.v8n2p54

\begin{abstract}
Early childhood is the most important time for establishing healthy eating habits, and of course during this period a mother can have a great influence in this regard. In this study explored what major factors influence the development children's food habits and whether the level of nutrition knowledge of parents has a material impact. This study involved 219 students (age renge, 3-5 years) and their parents in Osaka prefecture in Japan. Mothers were administered questionnaires that contained uestions pertaining to lifestyle, unidentifiable description of the children and their guardian's SRH along with 39 questions to assess the food-related habits and attitudes. The correlates of the nutrition-related knowledge level of mothers and the children's concern and respect for healthy eating were assessed using the Chi-square test, Fisher's exact test and the Mann-Whitney U test. The differences between high and low amount of parental knowledge and high and low non-cognitive skills towards food groups were also examined. Completed questionnaires were returned by 219 of the participants. Among the respondents, $50.7 \%$ were male, and $92.2 \%$ of the parents indicated that they get nutrition information from the Internet. We remarkably found that the level of mother's knowledge about nutrition had no statistically significant influence on how the children in this study understood food. However, chilren's concern and respect for food were significantly associated with their participation in various aspects of food preparation (help with washing and cooking of food) and presentation (help with setting up the table) ( $\mathrm{P}<0.05$, Mann-Whitney U test). Participation of Japanese kindergarten children in such activities was significantly associated with high level of food-related non-cognitive skills. Of note, the nutrition-related knowledge level of the mother showed no significant association with the attitude of their children towasds food.
\end{abstract}

Keywords: mother's nutrition knowledge, children's concern and respect for food

\section{Introduction}

Early childhood is the most important period for the inclusion of healthy eating habits and development of food preferences (Birch, 1979, Birch \& Sullivan 1991). Food habits established during early childhood are a key determinant of child development as well as the health of an individual throughout the life span (Scaglioni et at, 2008). Therefore, it is important that good attitudes towards nutrition and healthy eating habits are well established in the early childhood. In most, if not all, cultures, a mother has an enormous influence on her child's eating habits and food choices. For example, mothers who used chopsticks correctly affected their children's correct use of chopsticks (Osera et al, 2018a). In addition, high daily intake of soybean products by children was shown to be influenced by their mother's concern and positive attitudes towards the food habits of their children (Osera et al, 2016). In another study, the proportion of vegetables in the lunch box was shown to be related to mother's concern related to consumption of an appropriate mix of foods by their children to the preferences of the children (Osera et al, 2017). In addition, the development of children's food preferences involves a complex interplay of innate, familial and environmental factors (Cook, 2004). According to these earlier studies, a mother's actions and concerns about food is clearly an important element that shpes the attitude and behavior of children with regard to nutrition and food consumption. 
In this study we examined the influence of the nutrition-related knowledge level of the parents on the food-related behaviours of their children. According to the Knowledge-Attitude-Behavior (KAB) model, knowledge is important in order to shape decisions and to bring about behavior change. A version of the KAB model, i.e., Knowledge-Attitude-Practice, is an important theoretical construct for health education, which asserts that behavioural change is affected by knowledge and attitudes (Schneider \& Cheslock, 2003).

In addition, parent's knowledge of information maybe linked to good action. In a study by Jeong et al, all the heath belief model variables were shown to positively affect the customers' use of menu labels (Jeong \& Ham, 2017). Conversely, in another study, label use was related to engagement in some unhealthy behaviours in addition to healthy behaviours (Christoph et al, 2017).

We hypothesised that the higher the nutrition-related knowledge level of a mother, the 'better' will be her attitude towards healthy eating and that this will have a tangible influence on the food-related behaviour and habits of their children. To help address this question it was necessary to determine the knowledge level of parents related to childhood nutrition issues. In addition, our previous study suggested that children's food preferences are related to their concern and respect for food, and that children's non-cognitive skills food are associated with their life-style and daily food habits. A study difinded the children's non-cognitive skills as concerns about food and respect for food (Osera et al, 2018b). Accordingly this study attempted to measure parental nutrition knowledge and how this, or other factors, influenced children's attitudes and behaviours towards food and nutrition.

\section{Method}

\subsection{Design and Data Collection}

This cross-sectional study was conducted from September to December 2016. In total, 219 students, aged 3-5 years, and their guardians in Osaka prefecture, Japan participated in this study (Table 1). The mothers voluntarily opted to undergo the questionnaire survey.

The questionnaire was aimed to assess the lifestyle of children and their behaviour related to helping with cooking, as well as the parent's lifestyle and food habits. The questionnaire is a revised version of the questionnaire originally developed for our previous study (Osera et al, $2018 \mathrm{~b}$ ). The questionnaire consists of 11 questions related to lifestyle and food habits of children (e.g., wake-up time, bedtime, sleeping habits and frequency of eating breakfast), five questions related to mother's food habits and attitude towards food (e.g. frequency of eating breakfast, frequency of cooking, and use of Japanese food) and seven questions to assess the extent to which the children helped with cooking (e.g., washing rice, and setting up of table). 'Wake up time' was assessed by the questions 'What time do your children wake up?' and 'Concern about food' means 'Does your child have concerns about food?'

Both 4- and 5-point rating scales were used (higher scores indicated more positive food habits). For example, questions regarding 'concerns about food' utilised a 5-point rating scale ( $5=$ highly concerned; $4=$ moderately concerned; 3 = concerned, 2 = somewhat concerned and 1 = not concerned).

Based on our previous study, the non-cognitive skills towards food are defined as 'conerns about food' plus 'respect for food' (Osera et al, 2018 b). The maximum total score for concern for food and respect for food was 10, while the minimum score was 1 . We divided the respondents into two groups based on the mean scores.

\subsection{Amount of Information}

Nutrition-related knowledge level of mothers was addressed by 12 questions (Table 2). This instrument is a revised version of the one originally created for our previous study (Osera et al, $2018 \mathrm{~b}$ ).

\subsection{Statistical Analyses}

The Mann-Whitney $U$ test was used to evaluate the association between the independent variables and the knowledge level of the mother/food-related non-cognitive skills of children. In addition, $\chi^{2}$ tests were applied to assess the association between independent variables and SRH. For all analyses, $\mathrm{P}<0.05$ was considered statistically significant. Data processing and analyses were performed using the SPSS software for Windows, version 25.0 (IBM, New York, NY).

\subsection{Ethical Statement}

The participants were well-informed about the objectives and the methods of this study. Participation in the study was on a voluntary basis, with the option to withdraw at any point. Individual privacy was strictly protected throughout the investigation. This study was approved by the Osaka Seikei College Ethics Committee regarding Human Subjects. 
Table 1. Distribution of the study sample by sex, gender and working style

\begin{tabular}{llll}
\hline & Variables & Number & Percentage \\
\hline Sex & Male & 111 & 50.7 \\
& Female & 108 & 49.3 \\
\hline Grade & 3 years old class & 64 & 29.2 \\
& 4 years old class & 69 & 31.5 \\
& 5 years old class & 86 & 39.3 \\
\hline Occupation & House wife & 147 & 67.1 \\
& Part time job & 55 & 25.1 \\
& Full time & 11 & 5.0 \\
& Owner & 5 & 2.3 \\
& Other & 1 & 0.5 \\
\hline
\end{tabular}

\section{Results}

\subsection{Distribution of the Study Sample}

Complete responses to the questionnaire and characteristics of the study population are presented in Table 1. Of the 250 students and their guardians to whom the two questionnaires were sent, 219 returned both completed questionnaires. Of the 219 students, $49.3 \%$ were female and $50.7 \%$ were male; $29.2 \%$ were in the class for 3 year old children, while $31.5 \%$ and $39.3 \%$ were in the class for 4 - and 5- year old children, respectively.

\subsection{Relationship Between the Knowledge Level of Mother and Their And Children'S Concern and Habits Regarding Food}

In this study population, internet was the predominant source of nutritional information for a vast majority of parents $92 \%$ (Table 2). The mean score for knowledge level of parents in the study population was 3.7. Based on the knowledge level, we divided the respondents into two groups: 'low': score _ 3.9; 'high': score $\geq 4$.

Table 3 reports the relationship between the amount of mother's knowledge and how this relates to concern about food and food habits for both the mother and the children. Mothers of course have substantial information from each morning's frequency of cooking, the extent to which she explains traditional Japanese foods, and the eating habits of her children.

Table 2. How parents obtain information regarding nutrition

\begin{tabular}{ll}
\hline & Percentage \\
\hline Internet & 92.2 \\
Book & 66.1 \\
Family & 65.1 \\
TV & 59.6 \\
Friends & 43.1 \\
Letter from school & 14.2 \\
Package & 12.4 \\
Newspaper & 6.9 \\
Display advertising & 4.6 \\
Lecture of specialist's & 4.6 \\
Other & 2.3 \\
Health center & 0.6 \\
\hline
\end{tabular}


Table 3. Relationship between amount of mother's information and mother and children's concern and their food habits

\begin{tabular}{|c|c|c|c|c|c|c|c|c|c|}
\hline \multirow{3}{*}{ Children } & \multicolumn{8}{|c|}{ Amount of information } & \multirow{3}{*}{$P$ value } \\
\hline & \multicolumn{3}{|c|}{ Low } & \multicolumn{5}{|c|}{ High } & \\
\hline & $\mathrm{N}$ & Mean & \pm & SD & $\mathrm{N}$ & Mean & \pm & SD & \\
\hline Wake up time ${ }^{\S}$ & 105 & 3.30 & \pm & 0.60 & 114 & 3.41 & \pm & 1.32 & N.S. \\
\hline Sleeping habits $\$$ & 105 & 2.25 & \pm & 0.63 & 114 & 2.32 & \pm & 0.74 & N.S. \\
\hline Frequency of eating habits in the morning\# & 105 & 3.94 & \pm & 0.27 & 114 & 4.00 & \pm & 0.00 & 0.024 \\
\hline Frequency of eating habits with family in the morning\# & 105 & 3.70 & \pm & 0.75 & 114 & 3.80 & \pm & 0.63 & N.S. \\
\hline Talk about food ${ }^{\#}$ & 105 & 3.99 & \pm & 0.92 & 114 & 4.11 & \pm & 0.78 & N.S. \\
\hline Talk about taste ${ }^{\#}$ & 105 & 4.07 & \pm & 0.87 & 114 & 4.18 & \pm & 083 & N.S. \\
\hline Teeth brush ${ }^{\#}$ & 105 & 3.39 & \pm & 1.24 & 113 & 3.70 & \pm & 1.25 & N.S. \\
\hline Watch TV during dinner ${ }^{\#}$ & 105 & 2.24 & \pm & 1.40 & 114 & 2.22 & \pm & 1.40 & N.S. \\
\hline Respect for food ${ }^{\#}$ & 105 & 3.90 & \pm & 0.95 & 114 & 4.02 & \pm & 0.80 & N.S. \\
\hline Food-related concern ${ }^{\#}$ & 104 & 3.95 & \pm & 0.97 & 114 & 4.01 & \pm & 0.91 & N.S. \\
\hline Children who disliked food ${ }^{*}$ & 104 & 1.21 & \pm & 0.43 & 114 & 1.18 & \pm & 0.43 & N.S. \\
\hline \multicolumn{10}{|l|}{ Mother } \\
\hline Mother who disliked food ${ }^{*}$ & 104 & 1.55 & \pm & 0.57 & 114 & 1.57 & \pm & 0.56 & N.S. \\
\hline Frequency of eating habits in the morning ${ }^{\#}$ & 104 & 3.80 & \pm & 0.70 & 114 & 3.81 & \pm & 0.64 & N.S. \\
\hline Frequency of cook ${ }^{\#}$ & 104 & 3.29 & \pm & 1.02 & 114 & 3.71 & \pm & 0.88 & 0.002 \\
\hline Frequency of cooking Japanese traditional food ${ }^{\#}$ & 104 & 3.45 & \pm & 0.85 & 114 & 3.57 & \pm & 0.76 & N.S. \\
\hline Telling to Japanese traditional food ${ }^{\#}$ & 104 & 3.05 & \pm & 0.94 & 114 & 3.38 & \pm & 0.93 & 0.014 \\
\hline
\end{tabular}

The amount of mother's information low (0-3 point) vs. high (4-12) was assessed for significance using a Mann-Whitney U test.

This binary distinction was performed according to the median value.

*Guardians answered the children's disliked foods from 1 to 2 .

\$A four-point scale; the highest point indicates good habits.

\#A five-point scale; the highest point indicates good habits.

\subsection{Relationship Between Children'S Concerns and Respect and Mother and Children'S Concern and their Food Habits}

We observed a remarkable similarity between the level of non-cognitive skills in this study population with that measured in our previous study (7.6 and 7.9, respectively, on a 10-point scale). We divided the sample into two groups based on the level of these skills: high: score $\geq 8$; low: score $<8$. Table 4 presents the relationship of children's non-cognitive skills towards food with the following variables: children's sleeping habits, talk about food, talk about taste and the number of children who disliked food, mother's frequency of cooking and the frequency with which mothers told their children about traditional Japanese food. Tables 3 and 4 clearly demonstrate the strong relationship of the mother's knowledge level about good food habits and the food-related non-cognitive skills of children with two factors: the mother's frequency of cooking at home and how often she explained to the children about Japanese traditional food. 
Table 4. Relationship between children's concerns and respect and children and mother's food habits

\begin{tabular}{|c|c|c|c|c|c|c|c|c|c|}
\hline \multirow{3}{*}{ Children } & \multicolumn{8}{|c|}{ Children's concern and respect } & \multirow{3}{*}{$P$ value } \\
\hline & \multicolumn{3}{|c|}{ Low } & \multicolumn{5}{|c|}{ High } & \\
\hline & $\mathrm{N}$ & Mean & \pm & SD & $\mathrm{N}$ & Mean & \pm & SD & \\
\hline Wake up time ${ }^{\$}$ & 73 & 3.30 & \pm & 0.57 & 146 & 3.38 & \pm & 1.21 & N.S. \\
\hline Sleeping habits $\$$ & 73 & 2.16 & \pm & 0.65 & 146 & 2.35 & \pm & 0.70 & 0.035 \\
\hline Frequency of eating habits in the morning\# & 73 & 3.99 & \pm & 0.12 & 146 & 3.97 & \pm & 0.22 & N.S. \\
\hline $\begin{array}{l}\text { Frequency of eating habits with family in the } \\
\text { morning\# }\end{array}$ & 73 & 3.67 & \pm & 0.82 & 146 & 3.79 & \pm & 0.61 & N.S. \\
\hline Talk about food ${ }^{\#}$ & 73 & 3.88 & \pm & 0.93 & 146 & 4.14 & \pm & 0.80 & 0.035 \\
\hline Talk about taste ${ }^{\#}$ & 73 & 3.89 & \pm & 0.91 & 146 & 4.25 & \pm & 0.79 & 0.003 \\
\hline Teeth brush ${ }^{\#}$ & 73 & 3.30 & \pm & 1.30 & 145 & 3.68 & \pm & 1.22 & 0.043 \\
\hline Watch TV during dinner ${ }^{\#}$ & 73 & 2.12 & \pm & 1.44 & 146 & 2.28 & \pm & 1.37 & N.S. \\
\hline Children who disliked food ${ }^{*}$ & 73 & 1.14 & \pm & 0.45 & 145 & 1.23 & \pm & 0.42 & 0.025 \\
\hline \multicolumn{10}{|l|}{ Mother } \\
\hline Mother who disliked food ${ }^{*}$ & 73 & 1.52 & \pm & 0.60 & 145 & 1.58 & \pm & 0.55 & N.S. \\
\hline Frequency of eating habits in the morning ${ }^{\#}$ & 72 & 3.76 & \pm & 0.72 & 146 & 3.82 & \pm & 0.64 & N.S. \\
\hline Frequency of cook $^{\#}$ & 72 & 3.21 & \pm & 0.92 & 146 & 3.66 & \pm & 0.96 & 0.001 \\
\hline $\begin{array}{l}\text { Frequency of cooking Japanese traditional } \\
\text { food }^{\#}\end{array}$ & 72 & 3.36 & \pm & 0.70 & 146 & 3.59 & \pm & 0.84 & 0.028 \\
\hline Telling to Japanese traditional food ${ }^{\#}$ & 72 & 2.97 & \pm & 0.82 & 146 & 3.34 & \pm & 0.98 & 0.007 \\
\hline
\end{tabular}

The amount of concern and respect low (1-7 point) vs. high (8-10) was assessed for significance using a Mann-Whitney U test. This binary distinction was performed according to the median value.

*Guardians answered the children's disliked foods from 1 to 2 .

\$A four-point scale; the highest point indicates good habits.

\#A five-point scale; the highest point indicates good habits.

\subsection{Relationship Between Nutrition-Related Knowledge Level of Mother and Her Children'S Non-Cognitive Skills Related to Food and Its Preparation}

Remarkably, the level of mother's understanding of nutrition showed no association with children's food preparation-related behaviours (Table 5). On the other hand, children's non-cognitive skills towards food were significantly associated with children's cooking-related behaviours such as 'help cooking', 'help wash the food' and 'help set up the table' (Table 6). 
Table 5. Relationship between amount of mother's information and children's food related behaviours

\begin{tabular}{|c|c|c|c|c|c|c|c|c|c|}
\hline & \multicolumn{8}{|c|}{ Amount of mother's information } & \multirow{3}{*}{$P$ value } \\
\hline & \multicolumn{3}{|c|}{ Low } & \multicolumn{5}{|c|}{ High } & \\
\hline & $\mathrm{N}$ & Mean & \pm & SD & $\mathrm{N}$ & Mean & \pm & SD & \\
\hline Help set cooking & 105 & 1.98 & \pm & 1.39 & 114 & 2.01 & \pm & 1.24 & N.S. \\
\hline Help wash the rice $\mathrm{e}^{\#}$ & 105 & 1.91 & \pm & 1.15 & 114 & 2.10 & \pm & 1.28 & N.S. \\
\hline Help wash the food ${ }^{\#}$ & 103 & 2.07 & \pm & 1.24 & 114 & 2.36 & \pm & 1.34 & N.S. \\
\hline Help taste & 103 & 1.68 & \pm & 1.05 & 114 & 1.80 & \pm & 1.08 & N.S. \\
\hline Help set the table & 102 & 1.95 & \pm & 1.15 & 114 & 2.21 & \pm & 1.24 & N.S. \\
\hline Help bring dishes ${ }^{\#}$ & 104 & 3.89 & \pm & 1.08 & 114 & 4.04 & \pm & 1.06 & N.S. \\
\hline Help wash dishes ${ }^{\#}$ & 103 & 2.14 & \pm & 1.35 & 114 & 2.28 & \pm & 1.39 & N.S. \\
\hline
\end{tabular}

The amount of concern and respect low (0-3 point) vs. high (4-12 point) was assessed for significance using a Mann-Whitney U test. This binary distinction was performed according to the median value.

\#A five-point scale; the highest point indicates good habits.

Table 6. Relationship between children's concerns and respect and their food related act

\begin{tabular}{|c|c|c|c|c|c|c|c|c|c|}
\hline & \multicolumn{8}{|c|}{ Children's concern and respect } & \multirow{3}{*}{$\mathrm{P}$ value } \\
\hline & \multicolumn{3}{|c|}{ Low } & \multicolumn{5}{|c|}{ High } & \\
\hline & $\mathrm{N}$ & Mean & \pm & SD & $\mathrm{N}$ & Mean & \pm & SD & \\
\hline Help set cooking & 73 & 1.68 & \pm & 1.21 & 146 & 2.15 & \pm & 1.33 & 0.002 \\
\hline Help wash the rice ${ }^{\#}$ & 73 & 1.96 & \pm & 1.18 & 146 & 2.03 & \pm & 1.24 & N.S. \\
\hline Help wash the food ${ }^{\#}$ & 73 & 1.95 & \pm & 1.18 & 144 & 2.36 & \pm & 1.34 & 0.032 \\
\hline Help taste ${ }^{\#}$ & 73 & 1.64 & \pm & 1.09 & 144 & 1.79 & \pm & 1.06 & N.S. \\
\hline Help set the table $\mathrm{e}^{\#}$ & 73 & 1.79 & \pm & 1.11 & 143 & 2.24 & \pm & 1.22 & 0.005 \\
\hline Help bring dishes ${ }^{\#}$ & 73 & 3.92 & \pm & 0.89 & 145 & 4.00 & \pm & 1.15 & N.S. \\
\hline Help wash dishes ${ }^{\#}$ & 73 & 2.18 & \pm & 1.39 & 144 & 2.23 & \pm & 1.37 & N.S. \\
\hline
\end{tabular}

The amount of children's non-cognitive skill (concern about food plus respect for food) low (1-7 point) vs. high (8-10 point) was assessed for significance using a Mann-Whitney $U$ test. This binary distinction was performed according to the median value.

\#A five-point scale; the highest point indicates good habits.

\section{Discussion}

The knowledge level of mothers was related to children's frequency of eating behaviour in the morning, the mother's frequency of cooking and the extent to which she explained to her children about traditional Japanese food. This result suggests that mother's level of understanding about healthy nutrition was significantly related to the food-related behaviours of their children. On the other hand, a study by Vemula et al suggested that the effort to promote healthy food choices through the use of food labels is not completely effective (Vemula et al, 2013). In this context, our results suggest that in addition to increasing the amount of nutrition information received by parents, it is important to improve the quality of information and utilisation of this information by the mothers. In addition, children's non-cognitive skills towards food showed a significant association with their mother's frequency of cooking and the frequency with which she explained the various aspects of the nature of traditional Japanese food to them. These two factors were in turn significantly related to the nutrition-related knowledge level of the mother. These findings suggest that cooking at home by mothers is important for both children and mothers themselves, because it represents a medium through which nutritional information and values can be passed on to the kids. Also watching their mother's cooking behaviour may substantially heighten the children's appreciation and respect for food and healthy living in general. 
Approximately $67 \%$ of the respondent mothers in this study were homemakers. However, given the increasing trend of engagement of both parents in full time jobs, children are increasingly less likely to observe the cooking and food preparation efforts of their mothers at home in the future. This implies that nutrition education, especially regarding cooking, may be increasingly important for children in school. For example, the pilot programme 'LA Sprouts' was developed as a 12-week gardening, nutrition and cooking intervention course that targeted Latino youth in California. This experimental intervention resulted in increased preference for fruit and vegetable consumption and improved both cooking and gardening skills (Gatto et al, 2012). Therefore, with increasing engagement of mothers in full time employment outside the home, such programmes targeted at children may help improve the health of individuals throughout the life span. Such an intervention may possibly reduce the morbidity burden from lifestyle-related diseases such as heart disease and obesity and accrue considerable cost savings for the health systems.

In this study, food-related non-cognitive behaviours of Japanese kindergarten children were significantly associated with their domestic involvement in activities such as helping their mother with cooking, washing the food and setting the table. These results are consistent with those of a study by Weber Cullen et al. in which self-efficacy of children to eat fruits and vegetables showed a positive association with the actual consumption of these food items (Cullen et al, 2000). Fortunately, so, the children's own concerns pertaining to food and health, howsoever vaguely present, may help inculcate responsible dietary habits.

On the other hand, the level of a mother's knowledge about nutrition was not significantly related with the food-related behaviours of their children. This result suggests that involvement of children in cooking-related activities may inform their own concerns about health and the respect for food; this reflects the importance of actual physical involvement with meal preparation in order to achieve this type of result. Indeed, our previous study showed that holding of chopsticks correctly by the mother was directly related to their children doing likewise (Osera et al, 2018a). Underlying this behaviour, we found that the mother's concern about chopsticks was instrumental for her ability to hold them correctly (Osera et al, 2018a). Results of this study are quite similar to those of our previous study on this subject. More broadly, papers such as those by MacFarlane suggest that parents should be encouraged to listen to and consider the food preferences of their adolescents, provide a supportive family mealtime environment and offer healthy food at home (MacFarlane et al, 2010).

The level of a mother's nutritional sophistication was not directly connected to the food-related behaviour of their children in our study. These results suggest that childrens' own concerns directly influence their own behaviours. To change a child's behaviour, a mother should involve kids in the preparation of meals, explain the important aspects and respectfully listen to the feedback and the evolving attitudes of the child. In addition, for children, the broad family environmental context with respect to selection, preparation and consumption of food, including the influence of other people present at meals, is likely to have a major impact on the development of attitudes of the children towards nutrition.

A limitation of the current study is that it was a cross-sectional study and, therefore, could not demonstrate causal association. In our forthcoming project we intend to explore these issues further using retrospective and/or specific intervention methodologies. In this latter option, we intend to focus specifically on the mother's cooking and nutrition education, especially while she herself was at school. In addition, cooking schools for children have recently become quite popular; therefore, we would like to compare the nutrition-related behaviours of children who attend cooking school with those of children who do not. Further, we intend to assess the influence of such programmes on the lifestyle of children.

\section{Conclusions}

We found that for our group of Japanese kindergarten children their non-cognitive skills towards food (high concern and respect towards food) were significantly increased when they engaged at home with behaviours such as 'help cooking', 'help wash the food', and 'help set the table.' On the other hand, the depth of their mother's knowledge about nutrition was not significantly related to the children's actions regarding food.

\section{Acknowledgments}

We thank all the guardians and teachers at the kindergarten for their participation and cooperation throughout the study.

\section{Author Contributions}

TO, NT, HH, and NK developed the standardised protocol and the structured questionnaire. NT and HH conducted the research in preschool settings. TO drafted the manuscript. NK supported our study as supervisor. All authors critically revised the article for important intellectual content and approved the final manuscript. 


\section{References}

Birch, L. L. (1979). Preschool children's food preferences and consumption patterns. Journal of Nutrition Education, 11, 189-192. https://doi.org/10.1016/S0022-3182(79)80025-4

Birch, L. L., \& Sullivan, S. A. (1991). Measuring children's food preferences. Journal of School Health, 61, 212-214. https://doi.org/10.1111/j.1746-1561.1991.tb06015.x

Christoph, M. J., Loth, K. A., Eisenberg, M. E., Haynos, A. F., Larson, N., \& Neumark-Sztainer, D. (2017). Nutrition facts use in relation to eating behaviors and healthy and unhealthy weight control behaviors. Journal of Nutrition and Education Behavior, 17, 1499-4046. http://doi.org/10.1016/j.jneb.2017.11.01

Cook, L. (2004). The development and modification of children's eating habits. The development and modification of children's eating habits. Nutrition Bulletin, 29, 31-35. http://doi.org/10.1111/j.1467-3010.2003.00388.x

Cullen, K.W., Baranowski, T., Rittenberry, L., Cosart, C., Owens, E., Hebert, D., \& Moor, C. (2000). Socioenvironmental influences on children's fruit, juice and vegetable consumption as reported by parents: reliability and validity of measures. Public Health Nutrition, 3, 345-356. https://doi.org/10.1017/S1368980000000392

Gatto, N. M., Ventura, E. E., Cook, L. T., Gyllenhammer, L. E., \& Davis, J. N. (2012). LA Sprouts. A garden-based nutrition intervention pilot program influences motivation and preferences for fruit and vegetables in Latino youth. Journal of the Academy of Nutrition and dietetics, 112, 913-920. https://doi.org/10.1016/j.jand.2012.01.014

Jeong, J. Y., \& Ham, S. (2017). Application of the heatlh belief model to customers' use of menu labels in restaurants. Appetite. 14, 208-215. https://doi.org/10.1016/j.appet.2017.12.012

MacFarlane, A., Crawford, D., \& Worsley, A. (2010). Associations between parental concern for adolescent weight and the home food environment and dietary intake. Journal of Nutrition Education and Behavior, 42, 152-160. https://doi.org/10.1016/j.jneb.2008.11.004

Osera, T., Tsutie, S., Kobayashi, M., \& Kurihara, N. (2016). Using soybean products in school lunch for health education may improve children's attitude and guardians' knowledge in kindergarten. Journal of Child and Adolescient Behavior, 4, 1000310. https://doi.org/10.4172/2375-4494.1000310

Osera, T., Tsutie, S., Kobayashi, M., \& Kurihara, N. (2017). Evaluation of children's lunch box contents by photograph and their relationship with mothers' concern. Journal of food research, 6, 78-86. https://doi.org/10.5539/jfr.v6n1p78

Osera, T., Yamamoto, C., Senke, R., Kobayashi, M., Tsutie, S., \& Kurihara, N. (2018 a). Relationship between mothers and children on how to hold chopsticks and concerns about chopsticks in Japanese kindergarten. Journal of Japanese Society of Shokuiku, 12, 1, 19-25. https://doi.org/10.14986/shokuiku.12.1

Osera, T., Tsutie, S., Kobayashi, M., \& Kurihara, N. (2018 b). The relationship between children's non-cognitive skills toward food and their food habits in a cross-sectional study. Journal of food research, 7, 1-9. https://doi.org/10.5539/jfr.v7n2p1

Scaglioni, S., Salvioni, M., \& Galimberti, C. (2008). Influence of parental attitudes in the development of children eating behavior. Brithsh Journal Nutrition, S1, S22-25. https://doi.org/10.1017/S007114508892471

Schneider, B., \& Cheslock, N. (2003). Measuring results: gaining insight on behavior change strategies and evaluation methods for environmental education, museum, health, and social marketing program. San Francisco, CA: Coevolution Institute.

Vemula, S. R., Gavaravarapu, S. M., Mendu, V. V. R., Mathur, P., \& Avula, L. (2013). Use of food label information by urban consumers in India - a study among supermarket shoppers. Public Health Nutrition, 19, 2104-2114. https://doi.org/10.1017/S1368980013002231

\section{Copyrights}

Copyright for this article is retained by the author(s), with first publication rights granted to the journal.

This is an open-access article distributed under the terms and conditions of the Creative Commons Attribution license (http://creativecommons.org/licenses/by/4.0/). 